\section{Melanoma: eaten up inside}

\section{By Michael J. Haas, Senior Writer}

Autophagy's dual pro- and antiapoptotic roles make it hard to determine whether inducing the process could be a viable strategy to treat cancer. In melanoma, a team led by Spanish researchers thinks the answer is yes. The group created a complex composed of a double-stranded RNA mimetic and a cationic carrier that selectively induced autophagy and cell death in melanoma but not normal skin cells. ${ }^{1}$

The question is whether the complex can be given at low doses that bypass the known toxic effects of the dsRNA mimetic in humans.

Intra- and extracellular stresses can trigger autophagy, the process by which cells degrade their own cytosolic components to prolong cell survival and-in the case of cancer-favor tumor growth. By mechanisms that are not well understood, autophagy also plays a proapoptotic role in the immune system's clearance of infected cells.

Given melanoma's known resistance to proapoptotic chemotherapeutics, which trigger cell death by causing irreparable DNA damage, a team led by María Soengas, group leader of the melanoma laboratory at the Spanish National Cancer Research Centre (CNIO), set out to determine whether melanoma was sensitive to apoptosis triggered by the cells' protective autophagic response to stress.

First, the researchers screened chemotherapeutics and immunomodulatory agents for compounds that selectively induced autophagy and cell death in melanoma cells but not in normal skin cell lines. They identified the dsRNA mimetic polyinosine-polycytidylic acid (pIC) complexed with the cationic carrier polyethyleneimine (PEI) as a lead agent.

pIC is commonly used to mimic viral mRNA and thus viral infection in vitro; PEI is commonly used to deliver small interfering RNA to cells.

Compared with no treatment, the pIC-PEI complex decreased tumor size and tumor metabolic activity and increased progression-free survival (PFS) in three different mouse models of melanoma.

Microarray analysis and knockdown studies in melanoma cells showed that the complex triggered autophagy by upregulating a dsRNA sensor molecule called interferon induced with helicase C domain (1IFIH1; MDA5). This, in turn, upregulated a proapoptotic factor known as phorbol-12-myristate-13-acetate-induced protein 1 (PMAIP1; NOXA).
Neither molecule was upregulated in normal melanocytes treated with the complex, thereby confirming the complex's selective mechanism of action against melanoma, the team wrote in Cancer Cell.

In addition to CNIO, the group included researchers from Madrid's University Hospital 12 de Octubre, the University of Bonn and Virginia Commonwealth University.

\section{pIC and choose}

Multiple clinical trials of pIC to treat various non-melanoma cancers and viral infections have met with little success and have produced serious side effects. ${ }^{2-5}$ Thus, another cancer researcher suggested that the Cancer Cell authors determine whether the molecule behaves differently in melanoma or whether it's better to try to induce autophagy via different routes.

Larry Smith, CSO of Eleos Inc., told SciBX that the induction of autophagy was an interesting approach to treating melanoma but cautioned that he was "not optimistic that the particular agent the Soengas team tested is a good candidate."

"This pessimism extends to dsRNA as an inducer of autophagy in general" because dsRNA is known to have toxic effects, and the molecular factors involved in those toxicities appear to be the same factors that mediate pIC's induction of autophagy, he said. "Thus, I believe it may not be possible to separate the intended therapeutic effect and the toxic effects of a dsRNA inducer of autophagy."

To answer this question, Smith wanted to know whether the PIC-PEI complex was active against non-melanoma cancers in preclinical models. If it were, that would echo the previous studies of pIC complexes-which were successful in preclinical models but failed in the clinic - and thus suggest that the effects of pIC do not translate from mouse to human, he said. But if pIC-PEI were active only in melanoma models, Smith said that would suggest the complex might do better in the clinic.

Eleos' Aezea cenersen sodium (EL625), an antisense oligonucleotide against p53, is in Phase II testing to treat chronic lymphocytic leukemia (CLL) and small lymphocytic lymphoma.

Smith also expressed concerns about the safety of the PEI carrier. The molecule is less susceptible to in vivo degradation than a cell-penetrating peptide carrier, but the downside is that PEI could build up and have unknown effects, he said.

Soengas said her team observed no signs of toxicity in mice treated with the pIC-PEI complex.

She also noted that pIC's past failures in the clinic involved polylysine, not PEI, as a carrier. "PEI is much more efficient as a delivery agent than other carriers," she said. "We know of other labs using higher doses of PEI to deliver siRNA, also without apparent secondary effects."

Soengas added that her team did not yet know why PEI was such an efficient carrier for pIC. But the team thinks the mechanisms "may be 


\section{TARGETS \& MECHANISMS}

related to the specifics of the binding to the membranes and internalization in cells," she said.

Smith agreed that PEI appeared to be one of the better carriers but wanted to see it tested in patient-derived melanoma cells rather than the melanoma cell lines Soengas' team used in its in vitro and animal studies.

"I do not believe there are any data to show that polyethyleneimine can facilitate dsRNA uptake into melanoma cells freshly obtained from patients," he said. "Cell lines are substantially different in a number of regards and are not very predictive of what happens with clinical cancer."

Smith also wanted to see additional mechanistic studies that elucidate how autophagy is triggered in melanoma and how it kills the cells. "This could lead to an understanding of the requirements for novel agents that might produce this effect and have less toxicity" in humans than was previously found with pIC, he said.

Soengas said her team is already investigating the molecular mechanisms involved in autophagy-induced apoptosis in melanoma. The group's focus is "in the molecular links between dsRNA sensor [molecules] and autophagy."

The team is also testing other dsRNA mimetics as melanoma therapeutics and is interested in testing the pIC-PEI complex in other tumor types, Soengas said.

The findings reported in Cancer Cell are patented by the CNIO and are available for licensing. "We would definitely welcome partnerships with companies or agencies that would help us implement our results in the clinic," Soengas said.

Haas, M.J. SciBX 2(33); doi:10.1038/scibx.2009.1265

Published online Aug. 27, 2009

\section{REFERENCES}

1. Tormo, D. et al. Cancer Cell; published online Aug. 3, 2009; doi:10.1016/j.ccr.2009.07.004

Contact: María S. Soengas, Spanish National Cancer Research Centre (CNIO), Madrid, Spain

e-mail: msoengas@cnio.es

2. Robinson, R. et al. J. Natl. Cancer Inst. 57, 599-602 (1976)

3. Levine, A. \& Levy, H. Cancer Treat Rep. 62, 1907-1912 (1978)

4. Krown, S. et al. J. Biol. Response Mod. 4, 640-649 (1985)

5. Lampkin, B. et al. Cancer Res. 45, 5904-5909 (1985)

\section{COMPANIES AND INSTITUTIONS MENTIONED}

Eleos Inc., Omaha, Neb.

Spanish National Cancer Research Centre, Madrid, Spain

University Hospital 12 de Octubre, Madrid, Spain

University of Bonn, Bonn, Germany

Virginia Commonwealth University, Richmond, Va. 